\title{
A Study of GPS Carrier-Phase Time Transfer Noise Based on NIST GPS Receivers
}

\author{
Jian Yao and Judah Levine \\ National Institute of Standards and Technology, \\ Boulder, Colorado 80305, USA \\ and \\ JILA, University of Colorado, \\ Boulder, Colorado 80309, USA \\ jian.yao@nist.gov \\ judah.levine@nist.gov
}

To do a better time comparison between high-precision clocks (such as a Cesium-fountain clock and Hydrogen-maser clock), we want to study and eventually lower the GPS carrier-phase time transfer noise. The GPS carrier-phase time transfer noise comes from four sources: GPS satellite, GPS signal path, ground receiving equipment (receiver and antenna), and data-processing algorithm. This paper focuses on the noise introduced by the ground receiving equipment. At NIST, we have installed seven GPS receivers. All receivers have the same reference time, i.e., UTC(NIST). Three of them are connected to the same antenna. The other four are connected to four different antennas. This architecture enables us to study the time-transfer noise from the ground receiving equipment. We study both long-term (> 100 days) noise and short-term ( $<1$ day) noise. For the long-term noise, the time-transfer result using one receiver can vary from that using another receiver by up to $1.8 \mathrm{~ns}$, during 1.3 years. To achieve sub-nanosecond GPS timing accuracy, a careful monitoring of the time delays or a more frequent calibration is needed. For the short-term noise, we find that the common-clock difference between receivers using the same antenna is less noisy than that using two different antennas, at an averaging time of less than 0.5 hour. This indicates that the antenna and antenna cable contribute to the super-short-term noise of GPS carrier-phase time transfer significantly. In addition, the response to the GPS receiver's reference-time change is tested in this paper. The variation in the response can be up to \pm 350 ps. Last, this paper gives the best carrier-phase time transfer result we can currently achieve with the available equipment at NIST. The best frequency stability is $4.0 \times 10^{-16}$ at 3 hours, $1.1 \times 10^{-16}$ at 1 day, $4.0 \times 10^{-17}$ at 10 days, and $1.3 \times 10^{-17}$ at 48 days.

Key words: boundary discontinuity; carrier phase time transfer; GPS; long-term noise; precise point positioning (PPP); revised RINEX-shift; short-term noise; transient response.

Accepted: August 1, 2016

Published: August 8, 2016

http://dx.doi.org/10.6028/jres.121.017

\section{Introduction}

Global Positioning System (GPS) carrier-phase (CP) time transfer is a widely accepted high-precision time transfer method. This method provides much lower short-term ( $<1$ day) noise than other time transfer methods, such as GPS code-based common-view (CV) time transfer and Two Way Satellite Time and Frequency Transfer (TWSTFT) [1-5]. However, the short-term noise in GPS CP time transfer is still large, so that it is difficult to do a long-distance frequency comparison between two high-precision clocks (such as a Cesium Fountain clock and a Hydrogen-maser clock) for a time interval of less than 0.5 day (see [6]). The multi-receiver-single-antenna architecture at NIST enables us to study the short-term noise in GPS CP 
time transfer more thoroughly. To do the time comparison between two national metrology institutes (NMI), we need to know the time delay in GPS CP time transfer. Since 2015, the International Bureau of Weights and Measures (BIPM) has started to organize the calibration of some NMIs. Thanks to the calibration campaign [7-10], the time delay is well measured. However, little is known about the long-term change in the time delay. Ideally, the time delay is a constant. Practically, there must be some variation in the time delay. If the variation is big, the time comparison between two NMIs becomes inaccurate as time passes. Our earlier paper has studied the change in the time delay for about 100 days, based on a zerobaseline investigation (zero-baseline means that the physical distance between two receivers is almost zero, e.g., less than $1 \mathrm{~km}$ ) [11]. We further extend the time period from 100 days to 1.3 years in this paper.

Section 2 shows the experimental setup and introduces the data-processing methods. Section 3 presents the CP time transfer results using all NIST receivers, for about 1.3 years. Section 4 discusses the results from four perspectives: long-term noise, short-term noise, transient response to a reference-time change, and the possible best $\mathrm{CP}$ time-transfer performance. These discussions answer some major concerns about GPS CP time transfer and present a thorough study of the current performance of GPS CP time transfer. This provides a reference for the future improvement of GPS CP time transfer. Section 5 concludes the paper.

\section{Experimental Setup and GPS Data Processing}

At NIST, we had 7 GPS receivers running properly for most of Modified Julian Date (MJD) 56658 57133 (i.e., Jan. 01, 2014 - Apr. 21, 2015). They were NIS2, NISA, NISS, NIST, NISW, NISX, and NISY. All of them had the same reference time, i.e., UTC(NIST). NISA, NISS, and NISW were connected to the same antenna. The other four receivers were connected to four different antennas. The physical locations of all antennas are illustrated by Fig. 1. The maximum distance between two antennas was approximately $130 \mathrm{~m}$. The seven GPS receivers and their antennas were from several different manufacturers (see Table 1). Also, the NISA, NISS, NISW, and NIST receivers reside in a well-temperature-controlled room, while NIS2, NISX, and NISY are in the same big room which may have some temperature variation even within the room. ${ }^{1}$

In this paper, we achieve the GPS CP time transfer via three different data-processing methods: NRCan Precise Point Positioning (PPP) 1-day method, NRCan PPP 30-days method, and Revised RINEX-Shift (RRS) method. The NRCan PPP software package is developed by Natural Resources Canada [12]. Originally, it could only process the GPS data for a single day. We call it "NRCan PPP 1-day method" here. Similar to other PPP software (such as Atomium PPP [13] and Novatel GrafNav PPP), the NRCan PPP 1-day method has the problem of day boundary discontinuity in its time solution [14]. To mitigate this problem, people extend the length of data processing from 1 day to multi-days (e.g., 30 days) [15]. In this way, we no longer have the day boundary discontinuity. Instead, we have a discontinuity between two 30days data batches. We call this method "NRCan PPP 30-days method". To simplify things, we introduce a new term "data-batch boundary discontinuity", which occurs when we process different data batches. Both day boundary discontinuity and 30-days boundary discontinuity are actually "data-batch boundary discontinuity”. Typically, the data-batch boundary discontinuity is about $100 \mathrm{ps}-500 \mathrm{ps}$ [14-15]. The RRS method, invented in 2014, completely solves the problem of data-batch boundary discontinuity [16-17]. In the same year, we noticed that a GPS-data anomaly can lead to a discontinuity of a few hundred picoseconds [18]. We call this type of boundary discontinuity "anomaly boundary discontinuity (anomalyBD)". This discontinuity can typically be eliminated by repairing the anomalous GPS data with a highorder polynomial fitting, if the anomaly lasts for less than 40 min [18-19]. The current version of RRS has integrated the function of GPS-data repairing. Because of the ability to remove both data-batch boundary discontinuity and anomaly boundary discontinuity, RRS provides a continuous GPS carrier-phase time transfer result. Thus it is good for monitoring the small change in CP time transfer over a long-time interval. The performance of the RRS method can be found in [16, 20]. In [20], the short-term performance

\footnotetext{
${ }^{1}$ Certain commercial equipment, instruments, or materials are identified in this paper to foster understanding. Such identification does not imply recommendation or endorsement by the National Institute of Standards and Technology, nor does it imply that the materials or equipment identified are necessarily the best available for the purpose.
} 


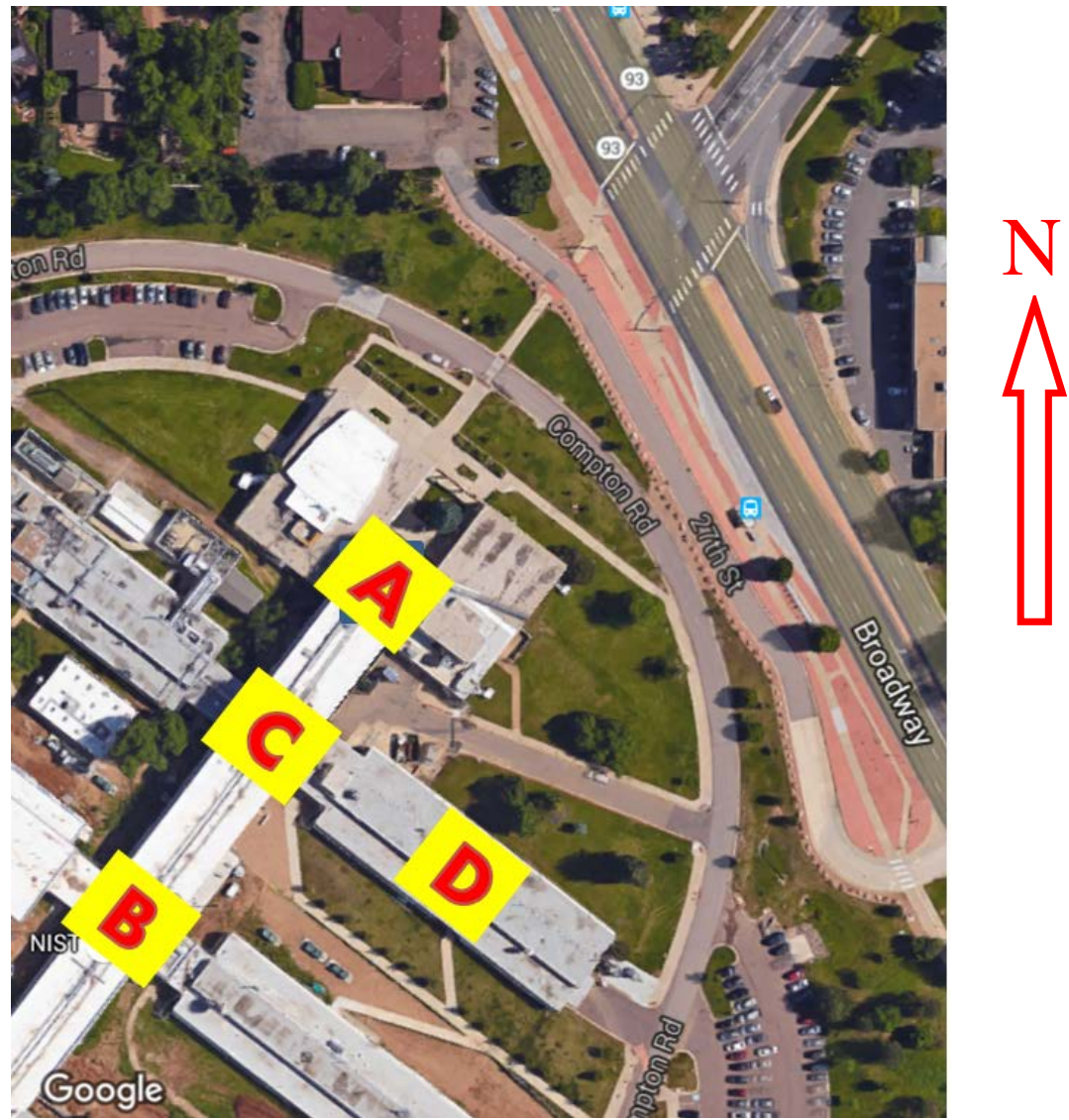

Fig. 1. Google map of NIST. The antennas of the NIS2, NISX, and NISY receivers were located in Zone A. The antennas of the NISA, NISS, NIST, and NISW receivers were located in Zone B. NISA, NISS, and NISW shared the same antenna. Zone C is a penthouse, and Zone $\mathrm{D}$ is a building.

Table 1. NIST receiver models and antenna models. Note, NISA, NISS, and NISW share the same antenna.

\begin{tabular}{|c|c|c|}
\hline Receiver Name & Receiver Model & Antenna Model \\
\hline NIS2 & Novatel OEM5 & NOV GNSS 750 (ChokeRing) \\
\hline NISA & Ashtech Z12T & Ash701945G_M (ChokeRing) \\
\hline NISS & SEPT Polarx3ETR & Ash701945G_M (ChokeRing) \\
\hline NIST & Novatel OEM4 & NOV 702 (Pinwheel) \\
\hline NISW & Novatel OEM5 & Ash701945G_M (ChokeRing) \\
\hline NISX & Novatel OEM4 & NOV 702 (Pinwheel) \\
\hline NISY & Novatel OEM5 & NOV GNSS 750 (ChokeRing) \\
\hline
\end{tabular}

of RRS was worse than that of NRCan PPP as indicated in [20]. A recent update of RRS shows that its short-term ( $<3$ hours) stability is now comparable to that of NRCan PPP [11]. This will also be shown later in this paper (see Fig. 5). To demonstrate that RRS, a relatively new method, is working correctly, we also provide NRCan solutions in this paper.

The three methods have the same settings. The cutoff elevation is 10 degrees. The mapping function of tropospheric delay is the "global mapping function (GMF)". We apply the same ocean tide loading to all NIST receivers. We only use GPS data, and do not use Glonass, Beidou, or Galileo data. The International GNSS Service (IGS) final products (SP3 and CLK) are used as the input files. 


\section{Results}

Here, we run the RRS data processing for all seven NIST receivers, for MJD 56658.000 - 57133.999 (i.e., Jan. 01, 2014 - Apr. 21, 2015). The results are shown by Fig. 2. We shift all curves so that they are zero at MJD 56658.000, for a better comparison. For the NISA and NISW receivers, since they started to record data properly on MJD 56690, we shift their time-transfer results (red curve and yellow curve) to be the same as the NISY's result (cyan curve) at 56690.000. Also, for the NISS receiver, it started to record data properly on MJD 56835. Thus we shift its result (black curve) to be the same as the NISY's result (cyan curve) at 56835.000. From Fig. 2, we can see that there are three sudden changes on MJD 56768, 56776, and 56818. These changes were caused by the change of reference-time signals. We can also see that the time differences between UTC(NIST) and the IGS final time scale via all receivers are almost the same. All curves have similar trends. However, there is still some small difference between curves. Figure 3 is enlarged from Fig. 2, which shows more details at around MJD 56736 (left plot) and MJD 57022 (right plot). We can see that the maximum time-transfer difference between two receivers is about $0.6 \mathrm{~ns}$ at MJD 56736 (i.e., Mar. 20, 2014), while the maximum time-transfer difference between two receivers is about 1.5 ns at MJD 57022 (i.e., Jan 01, 2015). This indicates that even if we have an ideal calibration at the beginning, the absolute GPS timing error can be as large as $1.5 \mathrm{~ns}$ after 1 year.

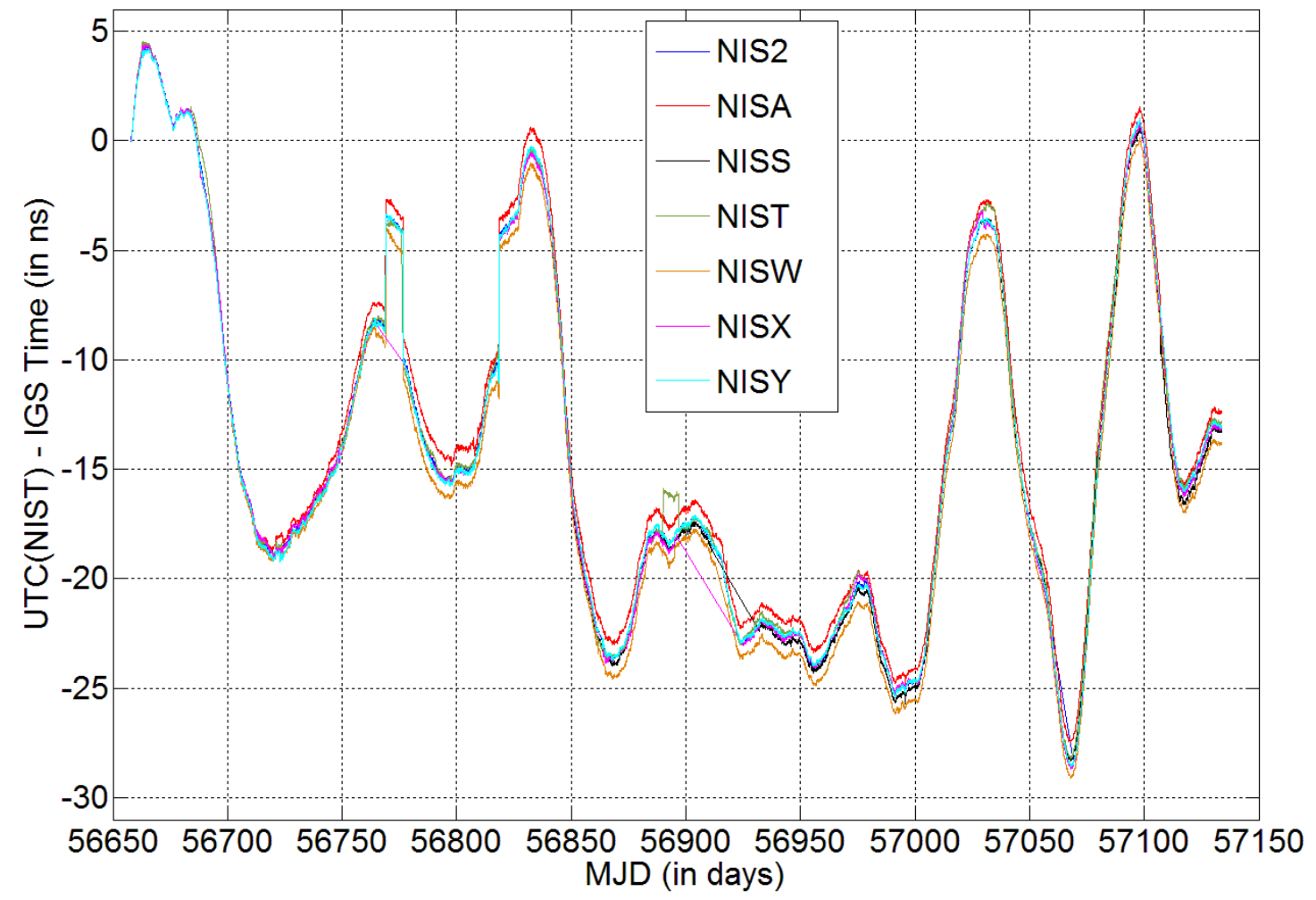

Fig. 2. Time difference between UTC(NIST) and IGS final time scale during MJD 56658.000 - 57133.999, using 7 NIST receivers. The curves are shifted to 0 ns at the epoch of MJD 56658.000, for a better comparison. Note, the NISA and NISW only have data from MJD 56690 on, and the NISS receiver only has data from MJD 56835 on. They are aligned with NISY at their first epochs. We have corrected the $50 \mathrm{~ns}$ ambiguity for $N I S X$, due to the $20 \mathrm{MHz}$ oscillator frequency, which may occur when there is a sudden referencetime change. 

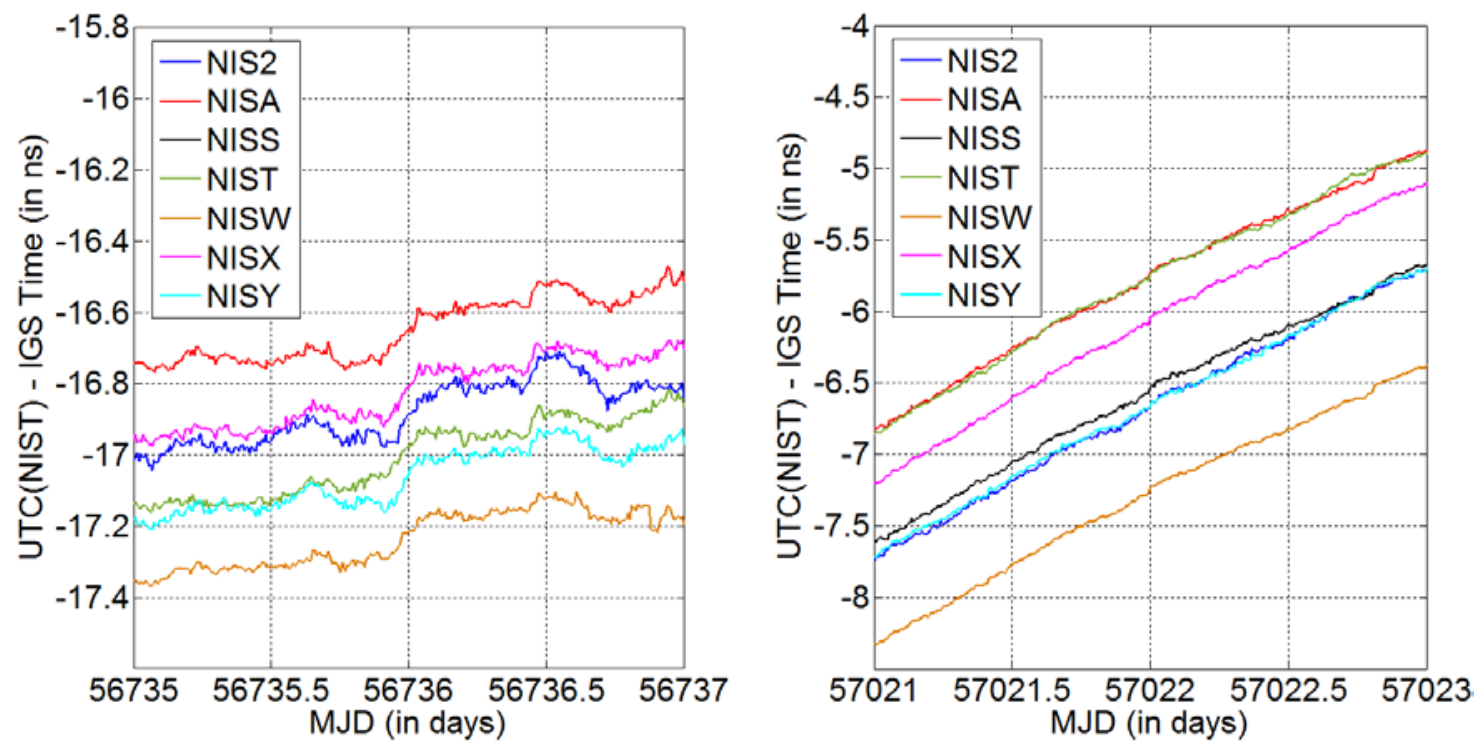

Fig. 3. Time difference between UTC(NIST) and IGS final time scale around MJD 56736 (left) and 57022 (right), using 7 NIST receivers. This figure is enlarged from Fig. 2. Note, the NISS data are not available in the left plot.

Another thing we want to address is that the curves in Fig. 2 do not diverge from each other linearly as time passes. In other words, there is no guarantee that one curve is above another curve all the time. As an example, at the beginning (i.e., MJD 56658.0), both NIS2 and NIST are 0. After 78 days (see left plot in Fig. 3), NIS2 is above NIST. However, after 1 year (see right plot in Fig. 3), NIS2 is below NIST. This means, the time delay for a receiver is dominated by a stochastic change, rather than a deterministically linear change.

\section{Discussions}

This section discusses four issues in GPS CP time transfer, based on the result in Sec. 3. They are longterm noise, short-term noise, transient response to a sudden reference-time change, and the best CP time transfer performance we can possibly achieve.

\subsection{Long-Term Uncertainty in GPS CP Time Transfer}

Section 3 has shown that different GPS receivers give different GPS CP time-transfer results. To see more details about the relative time change between two receivers, we show the time difference between two receivers in this section. The time difference between two receivers sharing the same reference time is also called "common-clock difference (CCD)" [21-23]. In principle, the time difference between two NIST receivers should be a constant. However, in practice, this is not exactly true as already indicated in Fig. 2.

The smallest long-term uncertainty between two NIST receivers using two different antennas is between NIS2 and NISY. Figure 4 shows the result using the RRS method (red curve). From the red curve, we find that the time difference between NIS2 and NISY changes by up to 300 ps (peak-to-peak) for the whole 476 days, if a few spikes are neglected. Although RRS aims at providing a continuous time-transfer result, there are still two obvious jumps in the red curve. One jump occurs at MJD 56768 which is due to the receiver's reference-time change. The other jump occurs at MJD 56863 which is due to a long data gap (> $40 \mathrm{~min})$. 


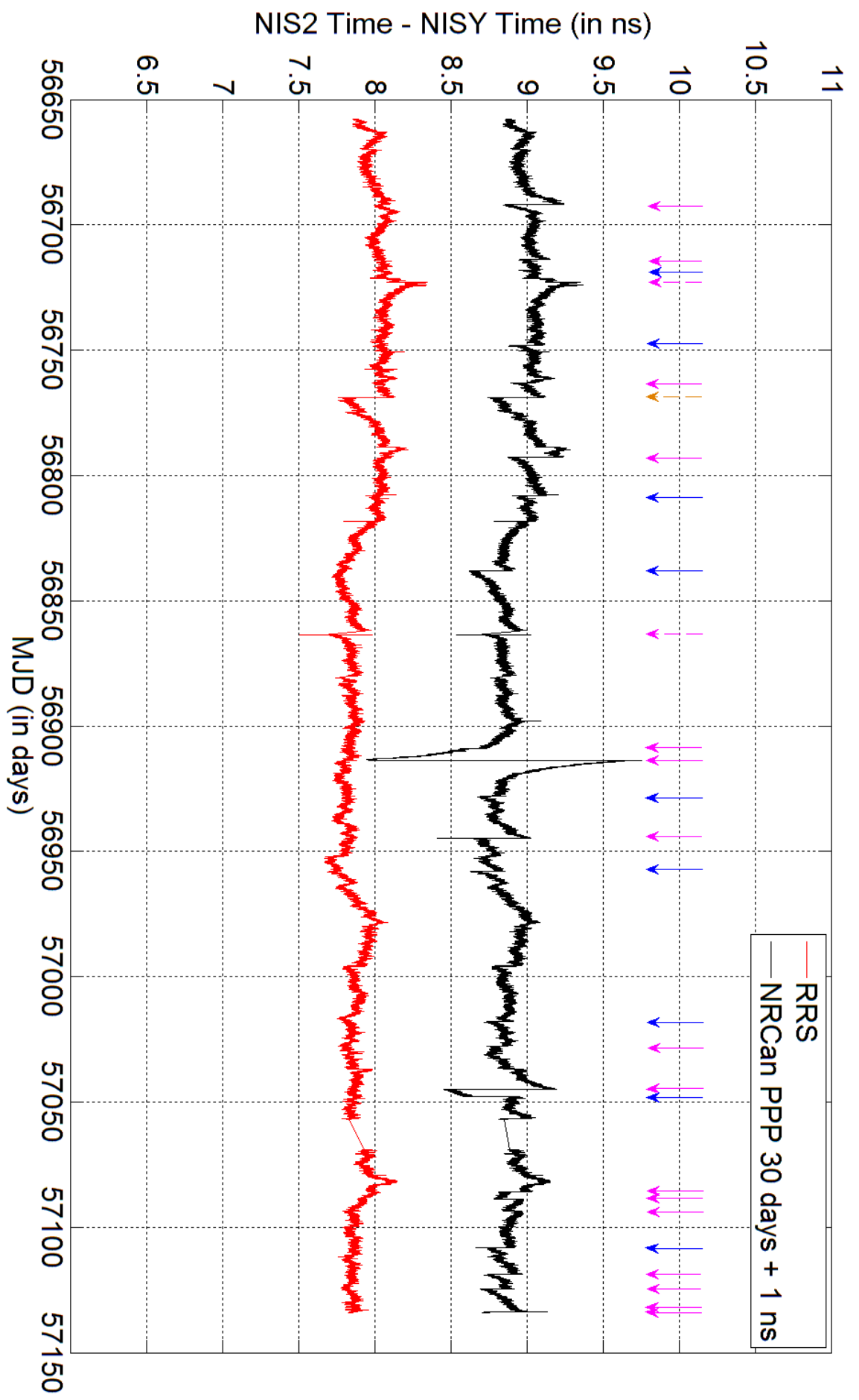

Fig. 4. Time difference between the NIS2 time (i.e., the UTC(NIST) via the NIS2 receiver) and the NISY time (i.e., the UTC(NIST) via the NISY receiver) during MJD 56658.000 - 57133.999, using the RRS method (red curve) and the NRCan PPP 30-days method (black curve). The NRCan PPP 30-days result is shifted by + $1 \mathrm{~ns}$, for a better comparison. There are 28 jumps in the NRCan PPP 30days result during the whole 476 days. The jumps are pointed out by arrows. The blue arrow indicates the 30-days boundary discontinuity; the magenta arrow indicates the anomaly boundary discontinuity; and the yellow arrow indicates the discontinuity due to the receiver's reference-time change. Dashed arrows are those boundary discontinuities that we cannot repair, typically because the anomalies last too long. 
Since RRS is a relatively new technique, we need to check if RRS is working properly. Thus, we compare the RRS result with the NRCan PPP 30-days result, to make the above result more convincing. The NRCan PPP 30-days result is shown by the black curve in Fig. 4. We can see that NRCan PPP 30-days has similar patterns to RRS. For example, both curves have a small bump at MJD 56665. Both have a peak at MJD 56975. This verifies that RRS is doing things right. From the black curve, we can also see that there are many jumps. The jumps of 30-days boundary discontinuity are pointed out by the blue arrows, and the jumps of anomaly boundary discontinuity are pointed out by the magenta arrows. There is also one jump due to the receiver's reference-time change on MJD 56768, which is labeled by the yellow arrow. Note, some jumps may look tiny in Fig. 4 because of the scale. However, if we enlarge the figure, these jumps are obvious.

Comparing the black curve with the red curve in Fig. 4, the advantage of RRS over NRCan PPP 30days is obvious. RRS removes most jumps successfully, which makes the RRS result more reliable than the NRCan PPP 30-days result. In terms of frequency stability, RRS is slightly better than NRCan PPP 30-days just because it removes jumps. As examples, the left and right plots of Fig. 5 present the frequency stability of “NIS2 - NISY”, during MJD 56865.000 - 57055.999 and during MJD 57069.000 - 57133.999, respectively. We can see that RRS provides better stability than NRCan PPP 30 days at $0.25-10$ days. Here, we would like to emphasize that after the recent updates in RRS, the short-term stability of RRS is the same as that of NRCan PPP 30 days. Another thing we need to mention is that an incorrect slope often accompanies a boundary discontinuity in the NRCan PPP 30-days result. This can lead to an error in frequency transfer near the discontinuity. As an example, there is a jump of $~ 350$ ps at MJD 56691.830 in the black curve of Fig. 4. To see more details near this jump, we enlarge Fig. 4 (see Fig. 6). Clearly, the slope within \pm 3 days of the jump is not correct. If we use NRCan PPP 30-days to do frequency transfer for MJD 56688.0 - 56691.8, the error in frequency transfer is approximately $4 \times 10^{-16}$. In contrast, RRS provides a correct slope because it removes the boundary discontinuity.

We also provide the NRCan PPP 1-day result (see the blue curve in Fig. 7), as a reference. From the blue curve, it is very hard to identify the pattern in the time difference between NIS2 and NISY, because the day boundary discontinuity occurs too often and obscures the actual features.
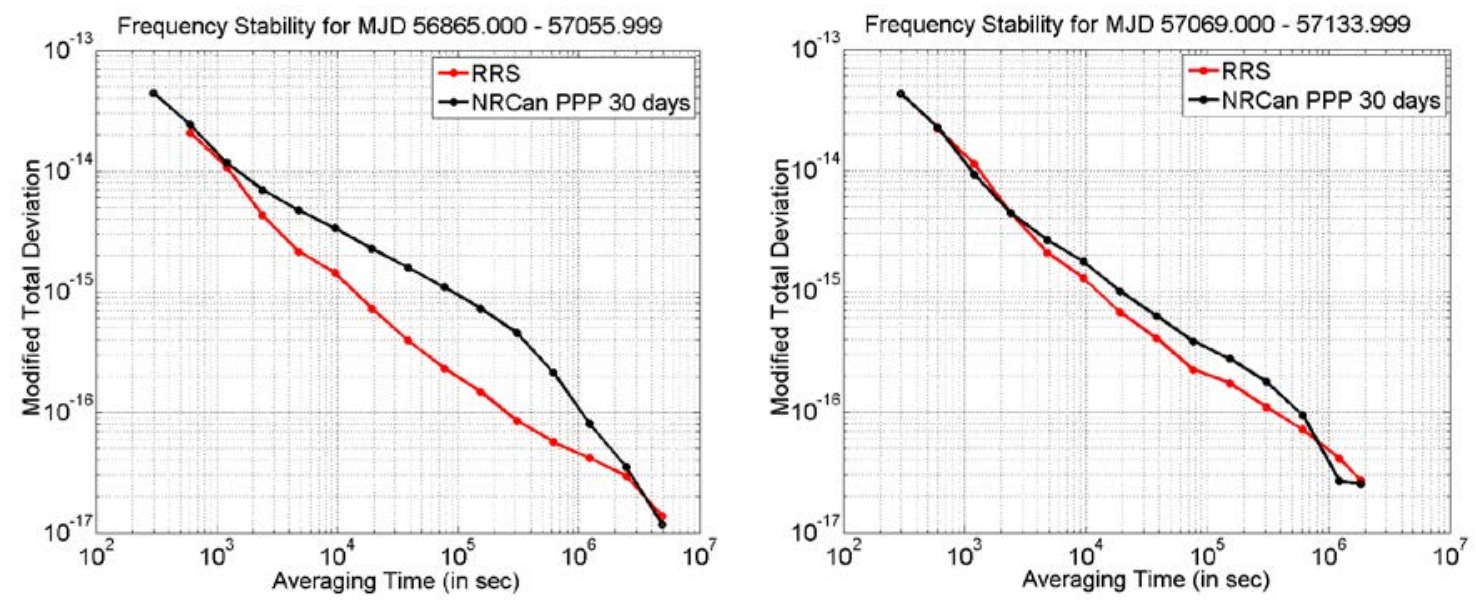

Fig. 5. Frequency stability of RRS (red) and NRCan PPP 30 days (black), for the double difference between NIS2 and NISY, during MJD 56865.000 - 57055.999 (left plot) and during MJD 57069.000 - 57133.999 (right plot). 


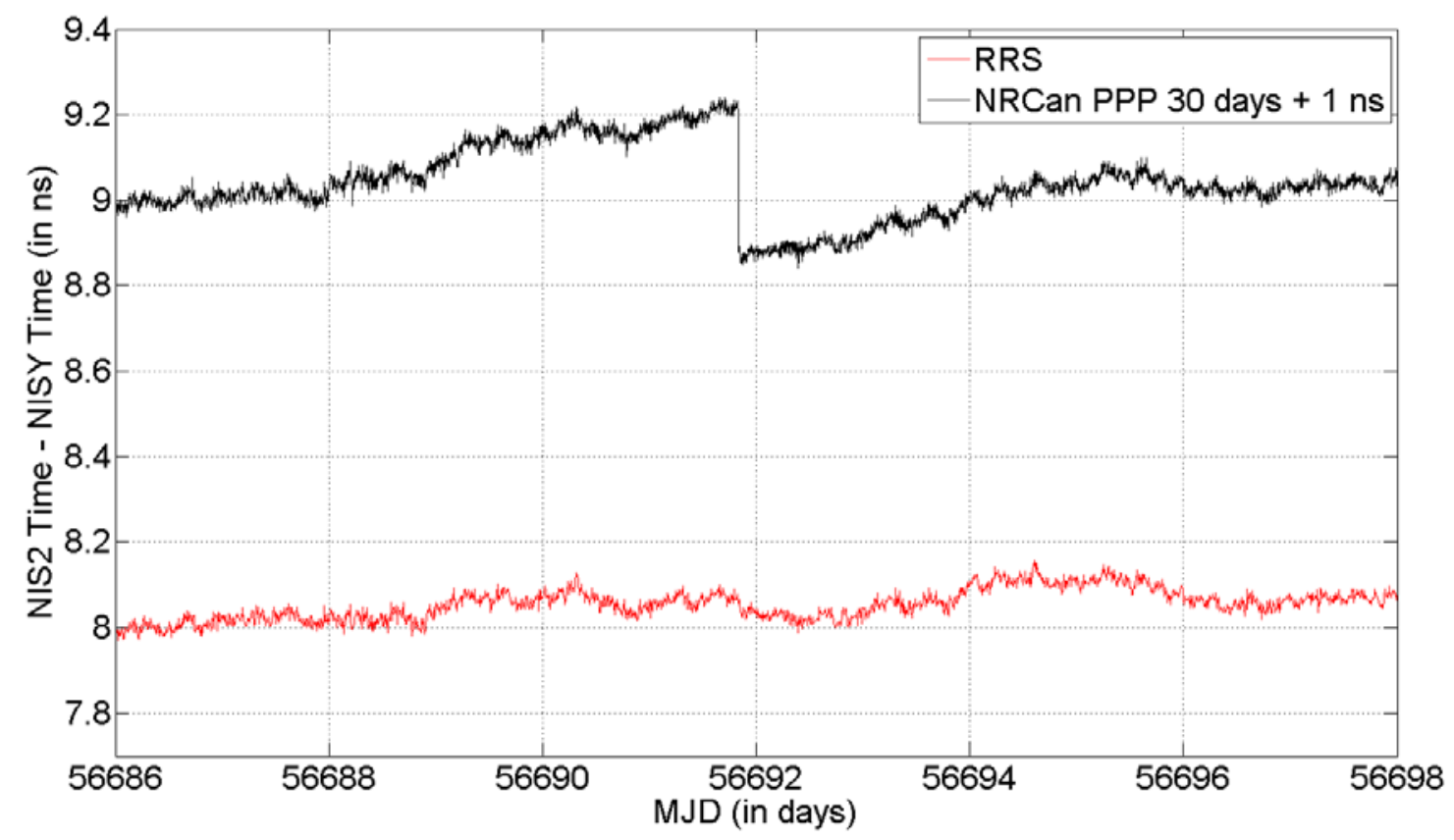

Fig. 6. Time difference between the NIS2 time and the NISY time around MJD 56691.830, using the RRS method (red curve) and the NRCan PPP 30-days method (black curve).

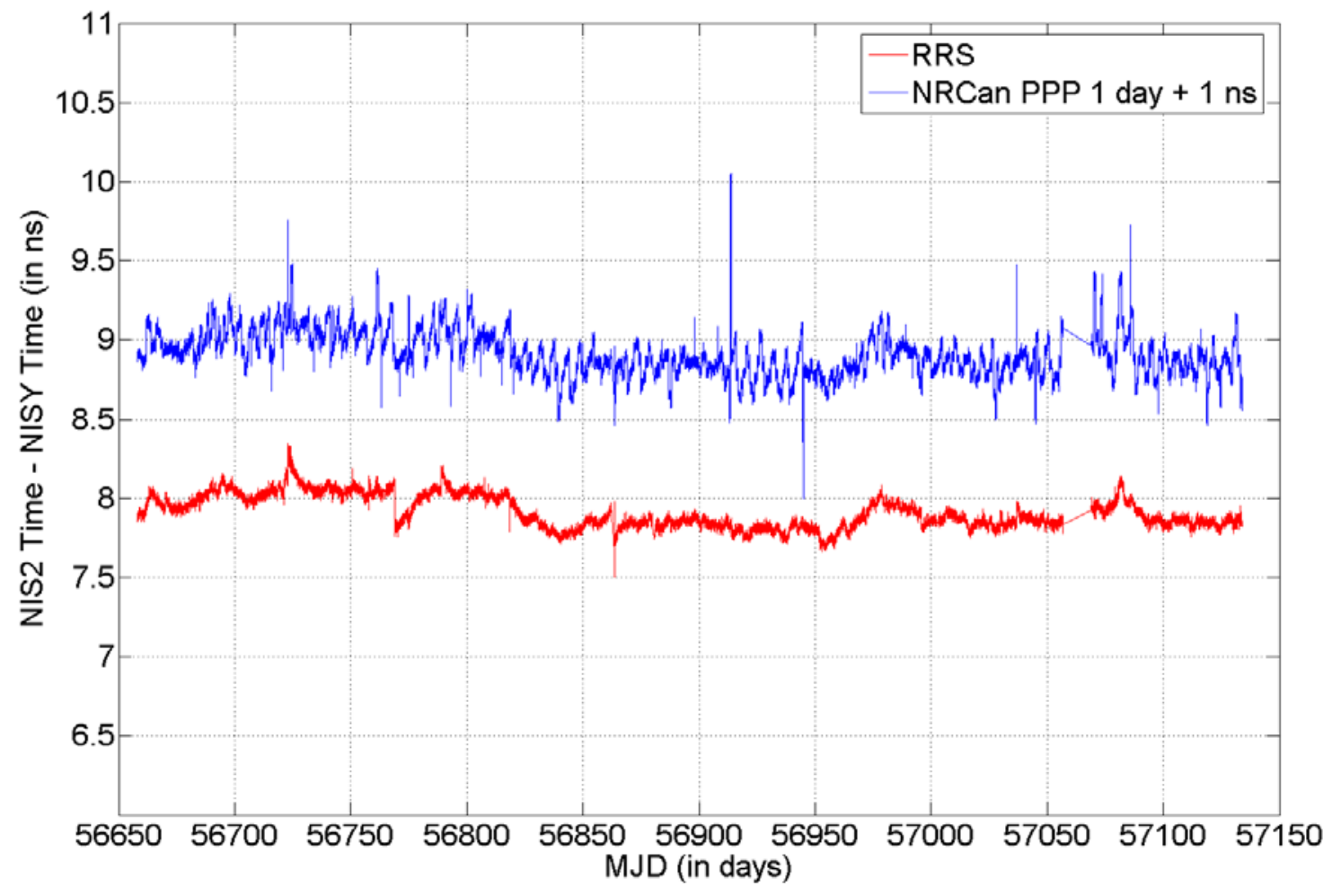

Fig. 7. Comparison of the RRS method and the NRCan PPP 1-day method. 
As we have mentioned earlier in this section, the link of "NIS2 - NISY" is more stable than other links over 1.3 years. We now study the long-term uncertainty of other links.

The NIST receiver has served as the primary NIST receiver for several years. Its long-term uncertainty is a significant concern for the time-transfer community, because it transfers the UTC(NIST) to BIPM and other NMIs. Some people also find that the NIST receiver has a saw-tooth behavior in its GPS CP timetransfer result [24-25]. In other words, the mean value of boundary discontinuities is far from 0 , for the NIST receiver. Here, we provide a 476-day comparison between NIST and NISY (Fig. 8). From the RRS result (red curve), we can see that the relative time change between NIST and NISY can go up to $\sim 1.7 \mathrm{~ns}$, even if we neglect the outlier at around MJD 56895. This big variation can be a problem for the GNSS (i.e., Global Navigation Satellite System) calibration, if sub-nanosecond accuracy is needed. For example, suppose we do the calibration during MJD 57078 - 57085, we find that the average time-difference between NIST and NISY is approximately 632.4 ns. However, starting from 57095, the time-difference between the two receivers is actually only $631.2 \mathrm{~ns}$, which is $1.2 \mathrm{~ns}$ from the calibration. Thus, the calibration is off by $1.2 \mathrm{~ns}$. To achieve sub-nanosecond accuracy, a real-time monitoring of cable and device delays or a more frequent calibration are needed. Another thing we want to address is that the red curve does not have a long-term trend. From the start point (i.e., MJD 56658.000) to the end point (i.e., MJD 57133.999), the overall change in the red curve is less than \pm 200 ps. Also, we do not observe an obvious yearly behavior in the red curve (the two big peaks occur at MJD 56691 and MJD 57082). Last and most importantly, we do see the saw-tooth behavior in the NRCan PPP 30-days result, especially during MJD 56800 - 56850. RRS successfully removes the saw-tooth behavior, except the jump on MJD 56818 due to the reference-time change. People have proposed that the saw-tooth behavior may come from the code-phase bias [25]. To solve the saw-tooth behavior, people suggest to contact the manufacturers [24-25]. From this example, we can see that RRS is another solution to the saw-tooth behavior, which does not require the contact of manufacturers and is also easier for people to implement.

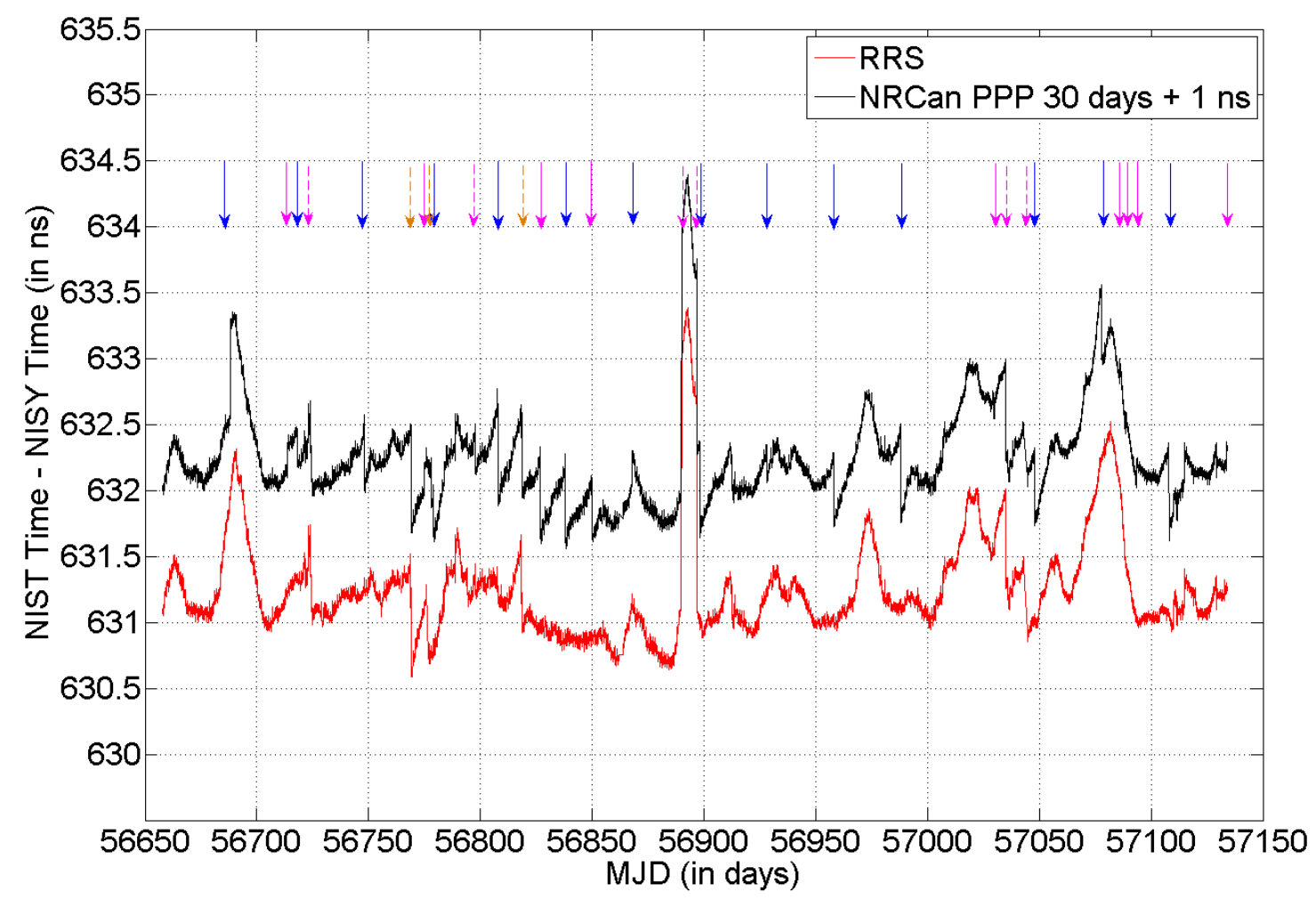

Fig. 8. Time difference between the NIST time and the NISY time during MJD 56658.000 - 57133.999, using the RRS method (red curve) and the NRCan PPP 30-days method (black curve). The NRCan PPP 30-days result is shifted by + 1 ns, for a better comparison. 
Next, we show the long-term change of two other links, "NISX - NISY" (top plot of Fig. 9) and "NISW - NISY" (bottom plot of Fig. 9). The relative changes between NISX and NISY, and between NISW and NISY are both less than 1 ns (peak-to-peak) during the whole time period, if the spikes are neglected. In addition, from the black curve of the top plot, we can see that there seems to be some saw-tooth behavior in the NISX receiver. The behavior is quite obvious during MJD 56800 - 56850. RRS successfully removes the saw-tooth behavior during MJD 56800 - 56850.
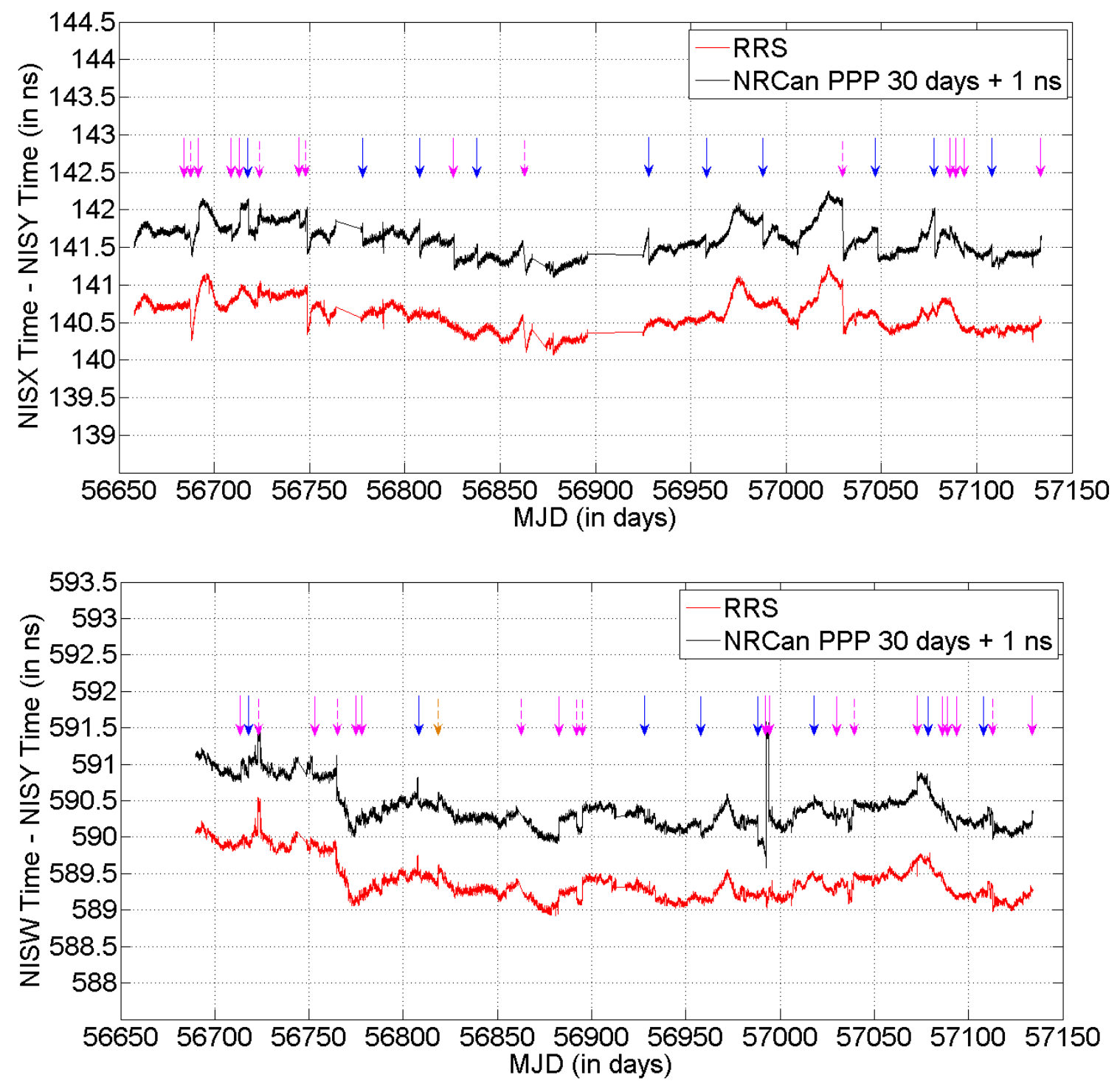

Fig. 9. Time difference between the NISX time and the NISY time during MJD 56658.000 - 57133.999 (top) and between the NISW time and the NISY time during MJD 56690.000 - 57133.999 (bottom), using the RRS method (red curve) and the NRCan PPP 30-days method (black curve). 
The above discussion shows the time difference between receivers using different antennas. However, we do not know the time difference between receivers using the same antenna. To answer this concern, we show the time difference between NISW and NISA, and between NISA and NISS, using the RRS method (see Fig. 10). Note, NRCan PPP 30-days has more jumps than RRS and thus we do not show its result here. According to the blue curve in Fig. 10, the time difference between NISW and NISA varies by up to $1.8 \mathrm{~ns}$ during the whole 444 days. In contrast, the time difference between NISA and NISS only changes by up to 300 ps during 299 days. Thus, the long-term change between receivers using the same antenna can be either large or small. Comparing Fig. 10 with the time-difference results using different antennas (i.e., red curves in Figs. 4, 8, 9), we find that using the same antenna does not give an obvious improvement over the longterm stability of GPS CP time transfer.

Looking into the blue curve of Fig. 10, there seem many jumps. However, if we enlarge the figure, most apparent "jumps" are not the actual jumps. Figure 11 is enlarged from Fig. 10, at around MJD 56716. We can see that the "jump” at 56716 in the blue curve of Fig. 10 is not a jump. Instead, it is a real change in

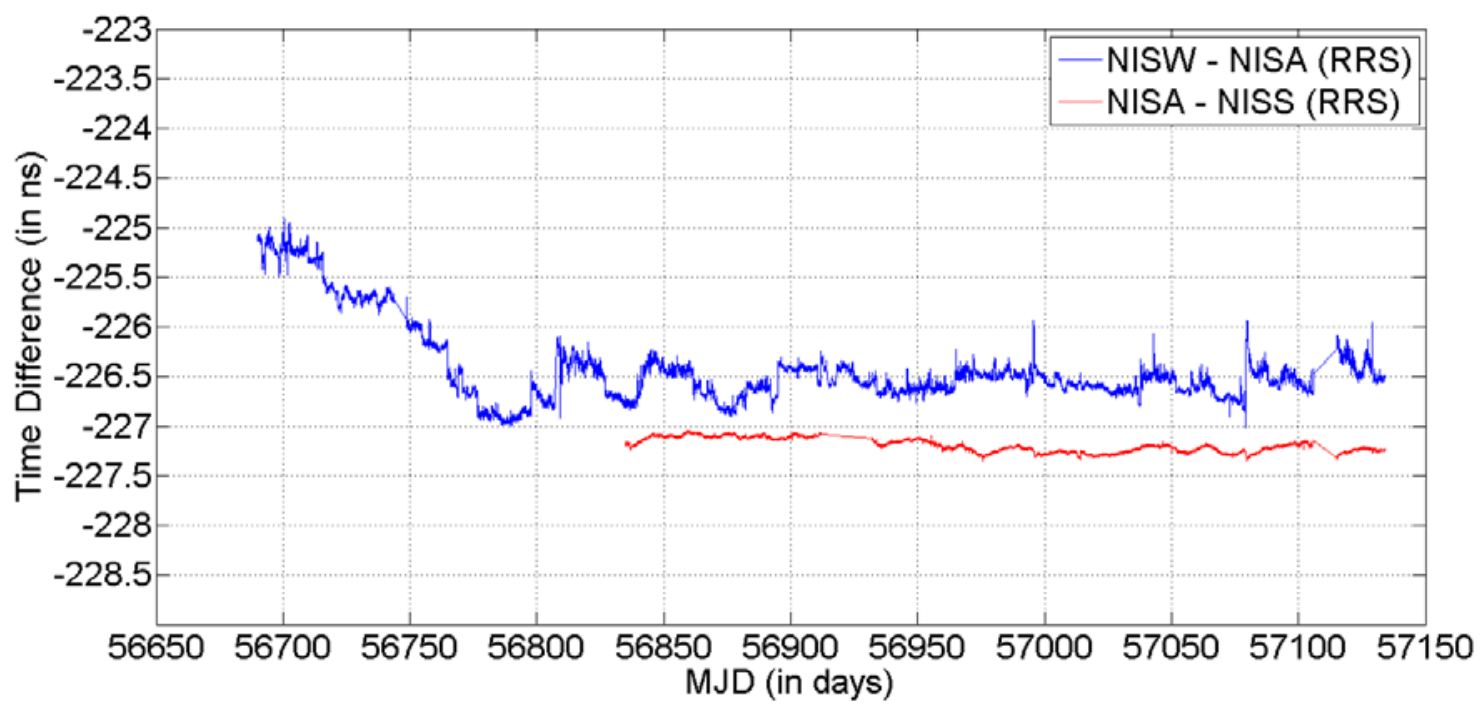

Fig. 10. Time difference between the NISW time and the NISA time during MJD 56690.000 - 57133.999 (blue), and between the NISA time and the NISS time during MJD 56835.000 - 57133.999 (red), using the RRS method.

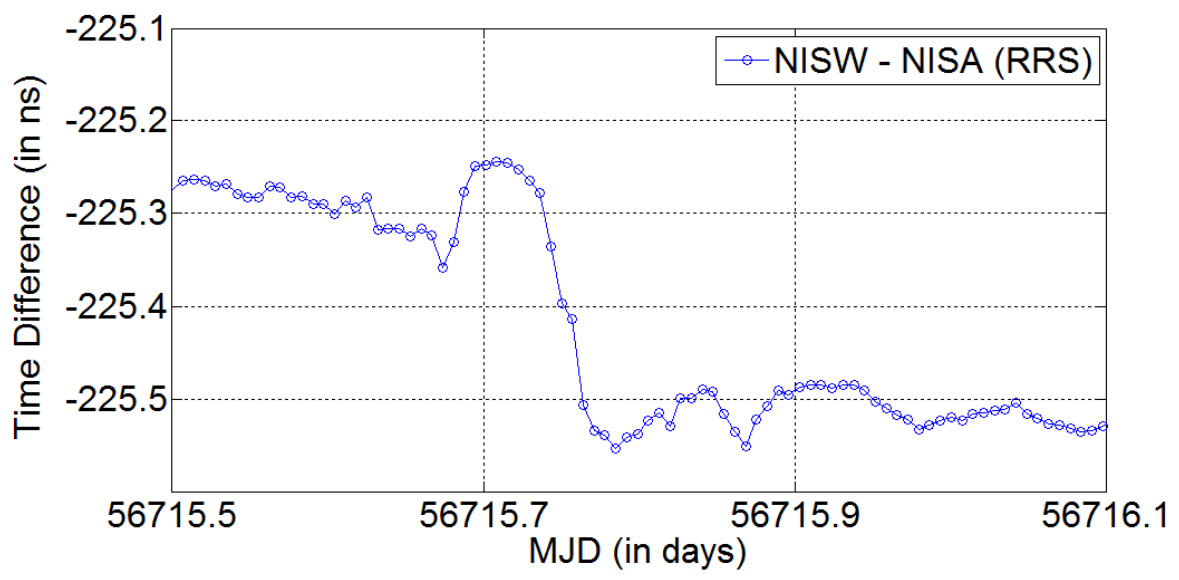

Fig. 11. Time difference between the NISW time and the NISA time, around MJD 56716. This figure is enlarged from Fig. 10. 
either NISW or NISA or both, within less than 2 hours. We currently do not understand why these two GPS receivers behave so differently for such a short time. More interestingly, NISW has good short-term match with other Novatel receivers (NIS2, NIST, NISX, and NISY), while NISA (Ashtech receiver) has good shortterm match with NISS (Septentrio receiver). Thus, the behavior in Fig. 11 could be an issue related to the manufacturers.

To conclude this section, the smallest long-term uncertainty in GPS CP time transfer we can achieve is 300 ps (peak-to-peak) during 476 days. However, for most cases, the long-term uncertainty can be as large as 1.8 ns (peak-to-peak) during 476 days. This poses a challenge to the GPS/GNSS calibration, if sub-nanosecond GPS timing accuracy is needed.

\subsection{Short-Term Noise in GPS CP Time Transfer}

For many NMIs, the stability of their timescales (e.g., UTC(k)) is better than the stability of GPS CP time transfer, at an averaging time of less than half a day. Thus, the GPS CP time transfer sets a fundamental limit for a remote high-precision clock comparison, for an averaging time of less than 0.5 day. The common-clock experiment and the common-clock-common-antenna experiment done at NIST give us the opportunity of distinguishing the short-term noise sources in GPS CP time transfer. This study provides a reference for lowering the short-term noise in GPS CP time transfer.

Figure 12 shows the typical frequency stability of three links: “NIST - USN7”, “NISA - NISY”, and “NISA - NISW”. Note, USN7 is a GPS receiver at United States Naval Observatory (USNO). Because NIST and USN7 have different reference times (i.e., UTC(NIST) and UTC(USNO)), the noise in the reference time contributes to the black curve. However, typically, the noise of UTC(k) is smaller than or comparable to an active hydrogen maser. And the noise of a maser is much less than the noise of GPS CP time transfer for an averaging time of less than 6 hours [26]. Thus, the black curve during $600 \mathrm{~s}-6$ hours can represent

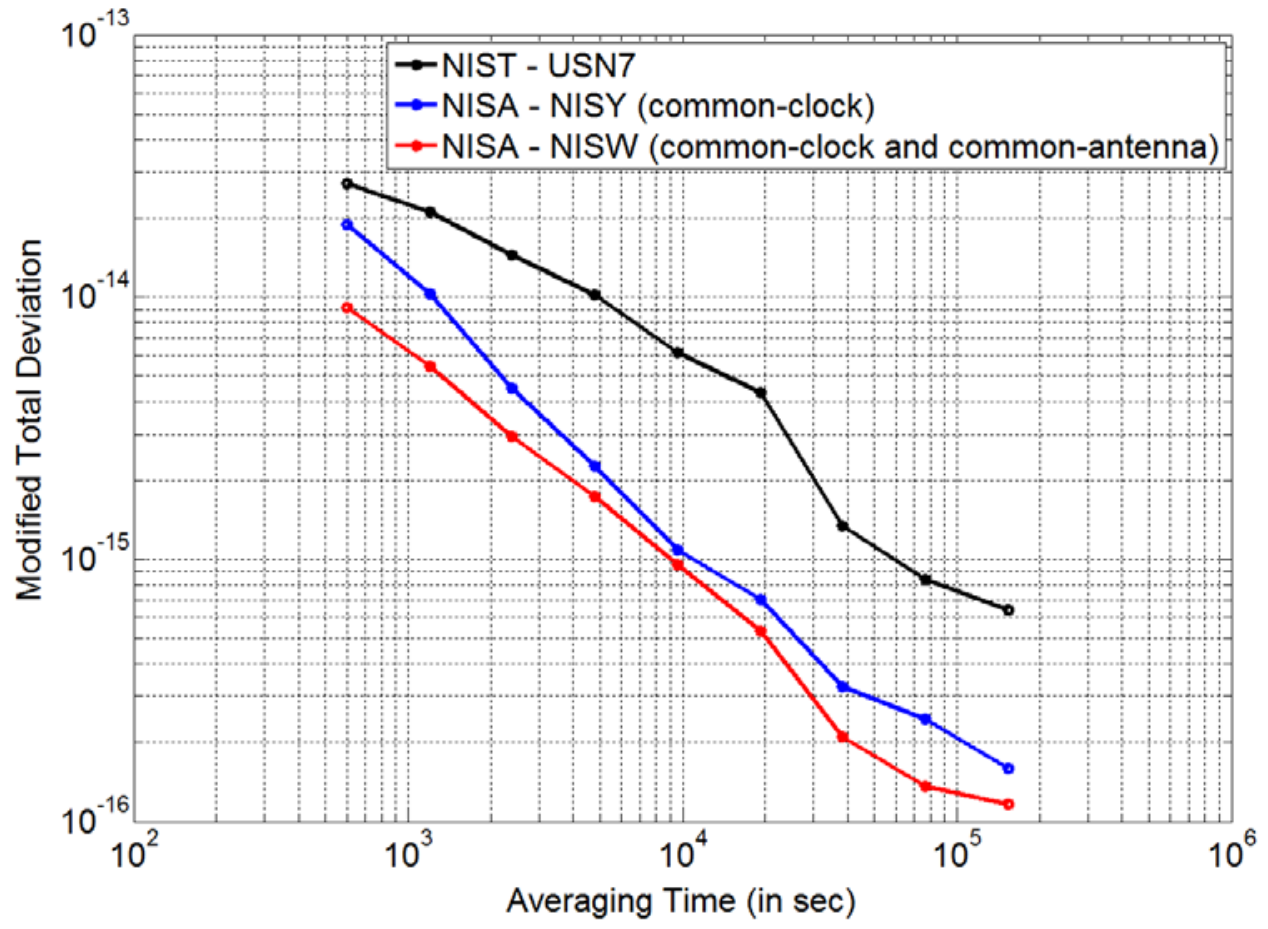

Fig. 12. Frequency stability of three links: "NIST - USN7” (black), "NISA - NISY” (blue), and "NISA - NISW” (red). The RRS results are used for all three curves. Note, NISA and NISW share the same antenna. Also, NISW and NISY have the same receiver model (see Table 1) and therefore their stability should be almost the same. 
the stability of GPS CP time transfer for non-zero baseline. Comparing the black curve with the blue curve, we can see that the noise of GPS CP time transfer for non-zero baseline is much bigger than that for zero baseline, at $0.5-6$ hours. This indicates that the noise from GPS-satellite parameters (such as position and time offset), and GPS signal path is much bigger than the noise from the ground-receiving equipment. Since the GPS-satellite parameters are estimated by IGS and the uncertainty is typically Gaussian distributed, the noise from GPS-satellite parameters should be averaged down quickly and becomes small at $0.5-6$ hours. Thus, the main reason why the black curve is much higher than the blue curve is that the signal-path noise is very big. The signal-path noise is composed of ionospheric noise and tropospheric noise. The first-order ionospheric noise is well cancelled because two frequencies are used. However, the second-order ionospheric noise cannot be cancelled since we do not use a triple-frequency GPS receiver. [27] shows that the second-order ionospheric noise can play some role in GPS positioning (up to $\sim 2 \mathrm{~cm}$ positioning error), especially when an extreme space weather event occurs. We currently do not know the impact of the second-order ionospheric noise on GPS timing (theoretically, the GPS timing should be correlated with the GPS positioning). A GPS CP time transfer experiment using triple-frequency GPS receivers can reveal this. In order to know the impact of tropospheric noise on GPS carrier-phase time transfer, a comparison between the time transfer using high-time-resolution (e.g., one data set each $30 \mathrm{~min}$ ) tropospheric parameters and the time transfer using low-time-resolution (e.g., one data set each 6 hours) tropospheric parameters is needed. In summary, the noise of GPS CP time transfer at $0.5-6$ hours mainly comes from the signal path. A time-transfer experiment using triple-frequency GPS receivers and a timetransfer experiment using high-time-resolution of tropospheric parameters can further reveal the contributions of ionospheric noise and tropospheric noise.

Comparing the blue curve with the red curve in Fig. 12, we can see that at 10 min, the frequency stability of the "NISA - NISY" link is 1.9 $\times 10^{-14}$, which includes the noise from both receivers, both antennas, and both antenna cables. In contrast, the instability of the "NISA - NISW" link is only $~ 9 \times 10^{-15}$, which only includes the noise from both receivers. Thus, the total noise from both antennas and both antenna cables is $1.7 \times 10^{-14}$. This means, the antenna and antenna cable noise is about twice as big as the receiver noise, at $10 \mathrm{~min}$. To achieve a good super-short-term ( $<0.5$ hour) stability of GPS CP time transfer, a careful selection of antenna and antenna cable is needed. In addition, the red curve is lower than the blue curve at $\sim 1$ day. Multipath or room temperature fluctuations may be the reason.

\subsection{Transient Response to the Reference-Time Change}

From time to time, the GPS receiver's reference-time may change. A good time-transfer technique should reflect the actual time change. As discussed in Sec. 5 of [20], RRS followed the time change within \pm 100 ps. Because we have several receivers at NIST, we can further study the statistics of the time-transfer error at a reference-time change. To lower the noise, we average the 2 hours of data before the referencetime change and the 2 hours of data after the reference-time change. Table 2 shows the result for the time change estimated by all NIST receivers, on MJD 56768. We can see that RRS provides similar results to NRCan PPP 30-days. Thus, both methods have comparable performance at a reference-time change. In addition, from the column "Delta using RRS", different GPS receivers have different estimations of the reference-time change. The mean value of the 6 estimations is $4.958 \mathrm{~ns}$. The values vary from the mean value within about $\pm 350 \mathrm{ps}$. The variation in the estimations comes from the fact that the estimation is composed of the actual reference-time change, and the boundary discontinuity which typically ranges from $-500 \mathrm{ps}$ to $+500 \mathrm{ps}$. One may want to compare the mean value of the 6 estimations with the actual reference-time change. Unfortunately, in this case, we do not have an actual measurement of the referencetime change. However, since the mean value of boundary discontinuities is near 0 , it is safe to assume that the mean value of the 6 estimations is close to the actual reference-time change. Based on the TWSTFT data, the estimation of the reference-time change is $5.079 \mathrm{~ns}$, which is quite close to the mean value 4.958 ns of the GPS receivers' estimations. This confirms the assumption that the mean value is close to the actual reference-time change. As a whole, the GPS CP time transfer is faithful to a sudden reference-time change within \pm 350 ps. 
Table 2. Transient response of RRS and NRCan PPP 30-days to a sudden reference-time change.

\begin{tabular}{|c|c|c|c|c|c|c|}
\hline $\begin{array}{l}\text { Receiver } \\
\text { (RCVR) }\end{array}$ & $\begin{array}{l}\text { "RCVR Time - } \\
\text { IGS Time” using } \\
\text { RRS (before } \\
\text { change) }\end{array}$ & $\begin{array}{l}\text { "RCVR Time - } \\
\text { IGS Time” using } \\
\text { RRS (after } \\
\text { change) }\end{array}$ & $\begin{array}{c}\text { Delta using } \\
\text { RRS }\end{array}$ & $\begin{array}{l}\text { "RCVR Time - } \\
\text { IGS Time" using } \\
\text { NRCan PPP } \\
30 \text { days (before } \\
\text { change) }\end{array}$ & $\begin{array}{l}\text { “RCVR Time - } \\
\text { IGS Time” using } \\
\text { NRCan PPP } \\
30 \text { days (after } \\
\text { change) }\end{array}$ & $\begin{array}{c}\text { Delta using } \\
\text { NRCan PPP } \\
30 \text { days }\end{array}$ \\
\hline NIS2 & $-511.601 \mathrm{~ns}$ & $-506.634 \mathrm{~ns}$ & $4.967 \mathrm{~ns}$ & $-511.595 \mathrm{~ns}$ & $-506.643 \mathrm{~ns}$ & $4.952 \mathrm{~ns}$ \\
\hline NISA & $296.396 \mathrm{~ns}$ & 301.444 ns & $5.048 \mathrm{~ns}$ & $296.397 \mathrm{~ns}$ & 301.407 ns & $5.010 \mathrm{~ns}$ \\
\hline \multicolumn{7}{|l|}{ NISS } \\
\hline NIST & $111.608 \mathrm{~ns}$ & $116.203 \mathrm{~ns}$ & $4.595 \mathrm{~ns}$ & $111.811 \mathrm{~ns}$ & 116.316 ns & $4.505 \mathrm{~ns}$ \\
\hline NISW & $69.745 \mathrm{~ns}$ & 75.017 ns & $5.272 \mathrm{~ns}$ & 69.735 ns & $74.979 \mathrm{~ns}$ & $5.244 \mathrm{~ns}$ \\
\hline NISX & $-378.395 \mathrm{~ns}$ & $-373.816 \mathrm{~ns}$ & $4.579 \mathrm{~ns}$ & $-378.426 \mathrm{~ns}$ & $-373.734 \mathrm{~ns}$ & $4.692 \mathrm{~ns}$ \\
\hline NISY & $-519.706 \mathrm{~ns}$ & $-514.422 \mathrm{~ns}$ & $5.284 \mathrm{~ns}$ & $-519.680 \mathrm{~ns}$ & $-514.409 \mathrm{~ns}$ & $5.271 \mathrm{~ns}$ \\
\hline
\end{tabular}

\subsection{Best GPS Carrier-Phase Performance at NIST}

Last, we discuss the best GPS CP time-transfer performance that we can currently achieve with the available equipment at NIST. At NIST, we mainly have four high-precision time-transfer techniques: GPS common-view time transfer, GPS CP time transfer, TWSTFT, and optical-fiber time transfer. GPS common-view uses the code, instead of carrier-wave, which makes it less precise than GPS carrier-phase. TWSTFT has the diurnal oscillation of $0.5 \mathrm{~ns}-1 \mathrm{~ns}$, which causes an inferior performance to GPS carrierphase, for an averaging time of less than 1 day. In the future, we plan to install the software-defined radio TWSTFT which may help remove the diurnal oscillation [28]. The optical-fiber link is between NIST and Alternate Master Clock (AMC, Colorado Springs, CO) using precision time protocol (PTP). According to [29], the current performance of optical-fiber time transfer at NIST is not as good as GPS carrier-phase. Thus, GPS carrier-phase currently serves as the best short-term time-transfer technique at NIST. As we have discussed in this paper, even for the GPS carrier-phase, its performance still depends on the specific receiver we use. This section presents the best GPS carrier-phase time-transfer result at NIST, so that we know the experimental limit we can achieve.

As we have already seen in Sec. 4.1, the best links of GPS CP time transfer at NIST are "NIS2 - NISY" (Red curve in Fig. 4) and "NISA - NISS" (Red curve in Fig. 10). Here, we do the frequency analysis of these two links, during MJD 56865.000 - 57055.999 (191 days) and during MJD 56932.000 - 57078.999 (147 days), respectively (see Fig. 13). The reason why we choose these two time periods is that the data are almost continuously recorded during the two periods. A link noise is composed of the clock noise and the time-transfer noise. Because the clock noise is cancelled out in each link, the link noise indicated in Fig. 13 is actually the GPS CP time-transfer noise. From the red curve, we can see that the frequency stability of GPS CP time transfer is $1.5 \times 10^{-15}$ at 3 hours, $2.1 \times 10^{-16}$ at 1 day, $5.0 \times 10^{-17}$ at 10 days, and $1.3 \times 10^{-17}$ at 60 days. Comparing the red curve with the blue dash line, we find that this performance enables us to see the Cs fountain clock behavior after 20 min. Also, we can use the GPS CP time transfer to monitor a shortdistance (e.g., < $100 \mathrm{~km}$ ) hydrogen-maser/time scale behavior. Admittedly, the red curve is only for zero baseline or nearly-zero baseline. The CP time transfer performance for non-zero baseline is worse than that for the zero baseline (see the black curve in Fig. 12). With a high-resolution of troposphere and a triplefrequency receiver in the future, we may possibly achieve the red curve in Fig. 12 for any baseline.

The magenta curve in Fig. 13 indicates that if we can eliminate the impact of the antenna, the frequency stability of GPS CP time transfer for zero/nearly-zero baseline can be further improved. It can reach $4.0 \times 10^{-15}$ at 3 hours, $1.1 \times 10^{-16}$ at 1 day, $4.0 \times 10^{-17}$ at 10 days, and $1.3 \times 10^{-17}$ at 48 days. 


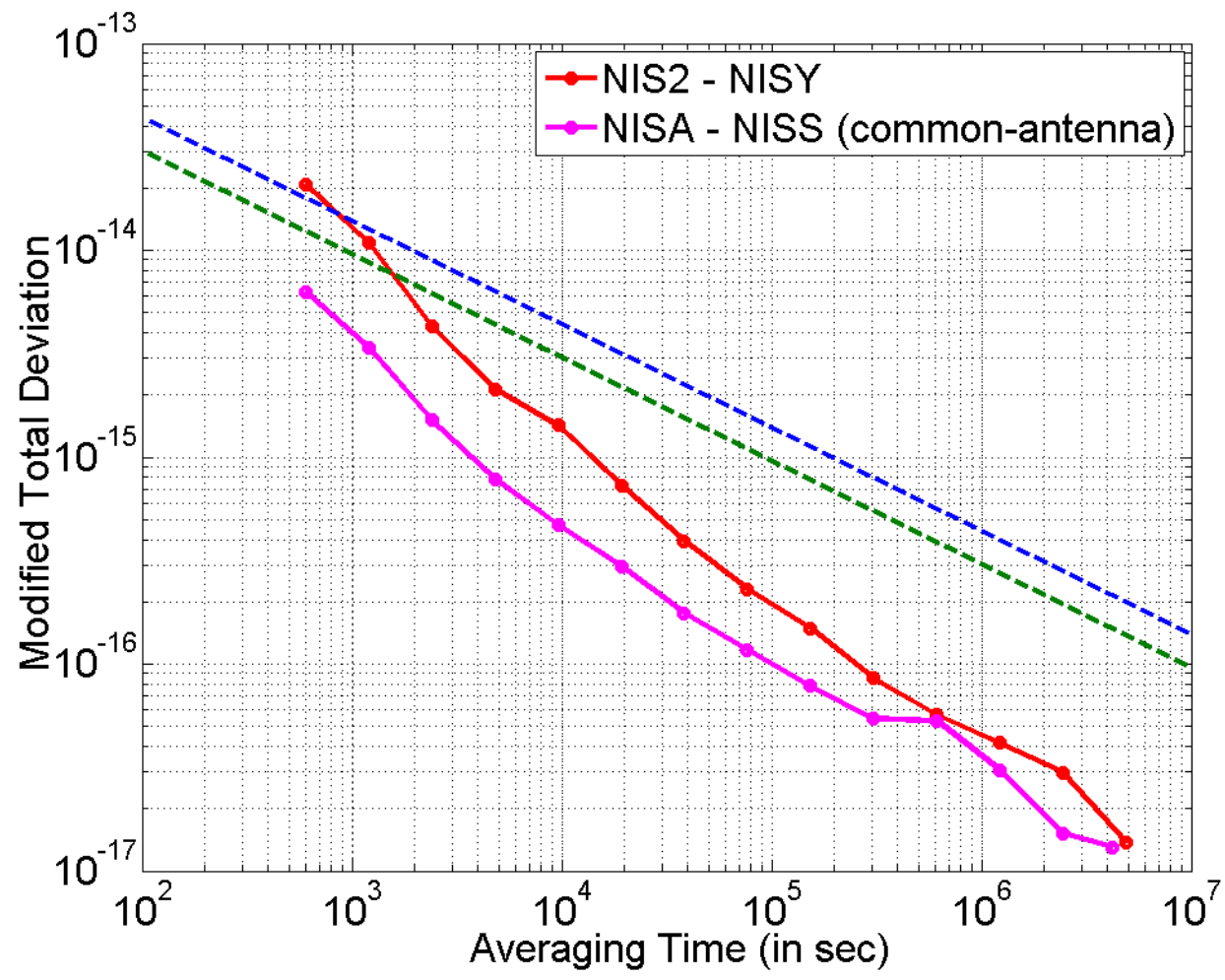

Fig. 13. Best GPS carrier-phase time transfer performance at NIST. The RRS results are used for both curves. Note, NISA and NISS share the same antenna. The red curve in this figure is the same as the red curve in the left plot of Fig. 5 . The green dash line is the frequency stability of a single Cesium fountain clock [30]. The blue dash line is the frequency stability of two Cesium fountain clocks compared to each other.

\section{Conclusions}

In summary, this paper studies the GPS carrier-phase time transfer from four perspectives: long-term ( $>100$ days) uncertainty, short-term ( $<1$ day) noise, transient response to a sudden reference-time change, and the possible best performance of GPS CP time transfer. We find that the difference in CP time transfer between two receivers can reach 1.8 ns (peak-to-peak) over 1.3 years, which poses a challenge for the GNSS calibration if sub-nanosecond GPS timing accuracy is needed. On the other hand, the smallest difference between two receivers over 1.3 year is 0.3 ns (peak-to-peak), which sets a fundamental lower limit for the long-term uncertainty in GPS timing accuracy. By comparing the zero-baseline link and the non-zero-baseline link, we know that the GPS signal-path has a significant impact on the GPS CP time transfer for an averaging time of $0.5-6$ hours. As for an averaging time of greater than 6 hours, we are unable to evaluate the impact of signal-path because the clock behavior starts to contribute. We also find that the antenna and antenna cable affect the performance of GPS CP time transfer quite significantly, at a super-short time ( $<0.5$ hour). Statistically, the GPS CP time transfer is faithful to the sudden reference-time change within approximately \pm 350 ps. Last, we have shown the best possible GPS CP time transfer performance. The frequency stability is $4.0 \times 10^{-16}$ at 3 hours, $1.1 \times 10^{-16}$ at 1 day, $4.0 \times 10^{-17}$ at 10 days, and $1.3 \times 10^{-17}$ at 48 days. This potentially enables us to see the short-distance (e.g., $<100 \mathrm{~km}$ ) high-precision clock behavior, after $0.5-1$ hour. 


\section{Acknowledgments}

The authors thank Francois Lahaye for sharing the NRCan PPP software. We also thank Marc Weiss for installing and maintaining the NIST receivers. Victor Zhang and Tom Parker are thanked for providing the TWSTFT data and many constructive suggestions. In addition, we thank Christine Hackman for some helpful discussions. IGS is gratefully acknowledged for providing GPS tracking data, station coordinates, and satellite ephemerides.

\section{References}

[1] Jiang Z, Dach R, Petit G, Schildknecht T, Hugentobler U (2006) Comparison and combination of TAI time links with continuous GPS carrier phase results. In Proceedings of the 2006 20th European Frequency and Time Forum (EFTF), pp 440447.

[2] Lin SY, Tseng WH, Lin HT, Feng KM, Piester D (2009) The comparison between TWSTFT and GPS time transfer result of PTB-TL LINK. In Proceedings of the 2009 IEEE International Frequency Control Symposium Joint with the 22nd European Frequency and Time forum, pp 1203-1205. http://dx.doi.org/10.1109/FREQ.2009.5168390

[3] Zhang V, Parker TE (2013) A study of the diurnal in the transatlantic TWSTFT difference. 2013 Asia-Pacific Time and Frequency Workshop.

[4] Levine J (2008) A review of time and frequency transfer methods. Metrologia 45(6):S162-S174. http://dx.doi.org/10.1088/0026-1394/45/6/S22

[5] Hackman C, Levine J, and Parker T (2006) A long-term comparison of GPS carrier-phase frequency transfer and two-way satellite time/frequency transfer. In Proceedings of the 38th Annual Precise Time and Time Interval Systems and Applications Meeting, pp 485-498.

[6] Yao J (2014) Chapter 4: Eliminating Day Boundary Discontinuity: RINEX-Shift algorithm. Continuous GPS Carrier-Phase Time Transfer, Ph.D. Thesis, University of Colorado at Boulder.

[7] Uhrich P, Rovera GD, Chupin B, Galindo J, Esteban H, Jaldehag K, Rieck C, Bauch A, Polewka T, Cerretto G, Fantino G, Piriz R (2015) Use of two traveling GPS receivers for a relative calibration campaign among European laboratories. In Proceedings of the 2015 Joint Conference of the IEEE International Frequency Control Symposium \& the European Frequency and Time Forum, pp 643-648. http://dx.doi.org/10.1109/FCS.2015.7138927

[8] Kun L, Aimin Z, Weibo W, Dayu N, Yuan G, Zhiqiang Y, Kejia Z, Yue Z, Kun L, Bo L (2014) New timekeeping system and its time link calibration at NIM. In Proceedings of the 2014 IEEE International Frequency Control Symposium, pp 1-4. http://dx.doi.org/10.1109/FCS.2014.6859896

[9] Lin SY, Huang YJ, Tseng WH (2015) Uncertainty evaluation of 2013 TL METODE link calibration tour. In Proceedings of the 2015 Joint Conference of the IEEE International Frequency Control Symposium \& the European Frequency and Time Forum, pp 226-229. http://dx.doi.org/10.1109/FCS.2015.7138829

[10] Zhang V, Parker T, Yao J (2015) Long-term uncertainty in time transfer using GPS and TWSTFT techniques. In Proceedings of the 2015 Joint Conference of the IEEE International Frequency Control Symposium \& the European Frequency and Time Forum, pp 723-727. http://dx.doi.org/10.1109/FCS.2015.7138943

[11] Yao J, Levine J "Long-term GPS carrier-phase time transfer noise: a study based on seven GPS receivers at NIST" to be published in Proceedings of the 2016 IEEE IFCS Conference.

[12] Kouba J Héroux P (2001) Precise Point Positioning Using IGS Orbit and Clock Products. GPS Solutions 5(2):12-28. http://dx.doi.org/10.1007/pl00012883

[13] Baire Q, Bruyninx C, Defraigne P, Legrand J (2010) Precise point positioning with Atomium using IGS orbit and clock products: first results. Bulletin of Geodesy and Geomatics 69(2-3):396-404.

[14] Yao J (2014) Section 2.6: Other PPP Software Packages. Continuous GPS Carrier-Phase Time Transfer, Ph.D. Thesis, University of Colorado at Boulder.

[15] Petit G (2009) The TAIPPP pilot experiment. In Proceedings of the 2009 IEEE International Frequency Control Symposium Joint with the 22nd European Frequency and Time forum, pp 116-119. http://dx.doi.org/10.1109/FREQ.2009.5168153

[16] Yao J, Levine J (2014) An improvement of RINEX-Shift algorithm for continuous GPS carrier-phase time transfer. In Proceedings of the 27th International Technical Meeting of The Satellite Division of the Institute of Navigation, pp 1253-1260.

[17] Yao J, Levine J (2013) A new algorithm to eliminate GPS carrier-phase time transfer boundary discontinuity. In Proceedings of the 2013 Precise Time and Time Interval Meeting, pp 292-303.

[18] Yao J, Levine J (2014) GPS measurements anomaly and continuous GPS carrier-phase time transfer. In Proceedings of the 46th Annual Precise Time and Time Interval Systems and Applications Meeting, pp 164-169.

[19] Yao J, Levine J, Weiss M (2015) Toward Continuous GPS Carrier-Phase Time Transfer: Eliminating the Time Discontinuity at an Anomaly. J Res Natl Inst Stan 120:280-292. http://dx.doi.org/10.6028/jres.120.017

[20] Yao J, Skakun I, Jiang ZH, Levine J (2015) A detailed comparison of two continuous GPS carrier-phase time transfer techniques. Metrologia 52(5):666-676. http://dx.doi.org/10.1088/0026-1394/52/5/666

[21] Jiang Z (2016) Final Report of the BIPM Pilot Study on UTC Time Link Calibration. In Proceedings of the 47th Annual Precise Time and Time Interval Systems and Applications Meeting, pp 20-26.

[22] Jiang Z, Matsakis D, Zhang V, Esteban H, Piestre D, Lin SY, Dierikx E (2016) A TWSTFT Calibration Guideline and the use of a GPS Calibrator for UTC TWSTFT link Calibrations. In Proceedings of the 47th Annual Precise Time and Time Interval Systems and Applications Meeting, pp 231-242. 
[23] Petit G, Kanj A, Loyer S, Delporte J, Mercier F, Perosanz F (2015) 1 x 10(-16) frequency transfer by GPS PPP with integer ambiguity resolution. Metrologia 52(2):301-309. http://dx.doi.org/10.1088/0026-1394/52/2/301

[24] Matsakis D, Jiang Z, Wu W (2015) Carrier phase and pseudorange disagreement as revealed by Precise Point Positioning solutions. In Proceedings of the 2015 Joint Conference of the IEEE International Frequency Control Symposium \& the European Frequency and Time Forum, pp 717-722. http://dx.doi.org/10.1109/FCS.2015.7138942

[25] Defraigne P Sleewaegen JM (2015) Correction for code-phase clock bias in PPP. In Proceedings of the 2015 Joint Conference of the IEEE International Frequency Control Symposium \& the European Frequency and Time Forum, pp 662-666. http://dx.doi.org/10.1109/FCS.2015.7138930

[26] Microsemi. MHM 2010 Active Hydrogen Maser. http://www.microsemi.com/products/timing-synchronization-systems/timefrequency-references/active-hydrogen-maser/mhm-2010-active-hydrogen-maser\#overview

[27] Elmas ZG, Aquino M, Marques HA, Monico JFG (2011) Higher order ionospheric effects in GNSS positioning in the European region. Ann Geophys 29(8):1383-1399. http://dx.doi.org/10.5194/angeo-29-1383-2011

[28] Huang YJ, Fujieda M, Takiguchi H, Tseng WH, Tsao HW (2016) Stability improvement of an operational two-way satellite time and frequency transfer system. Metrologia 53(2):881-890. http://dx.doi.org/10.1088/0026-1394/53/2/881

[29] Weiss M, Cosart L, Hanssen J, Hicks S, Chase C, Brown C, Allen C, Johnson P, Wiltsie G., Coleman D (2014) Ethernet Time Transfer Through a U.S. Commercial Optical Telecommunications Network. In Proceedings of the 46th Annual Precise Time and Time Interval Systems and Applications Meeting, pp 214-220.

[30] Jefferts SR, Heavner TP, Parker TE, Shirley JH (2007) NIST cesium fountains: current status and future prospects. In Proceedings of SPIE 6673, Time and Frequency Metrology, pp 667309. http://dx.doi.org/10.1117/12.734965

About the authors: Jian Yao is currently a research associate at NIST and the University of Colorado at Boulder, where he performs studies in GPS carrier-phase time transfer and time scale algorithm under the supervision of Dr. Judah Levine. In 2014, he received a Ph.D. degree in physics from the University of Colorado at Boulder.

Judah Levine is a NIST fellow and the leader of the Network Synchronization Project in the Time and Frequency Division at NIST. He is responsible for the design and implementation of the time scales AT1 and UTC(NIST). In addition, he designed and built the servers that support the Automated Computer Time Service (ACTS) and the Internet Time Service.

The National Institute of Standards and Technology is an agency of the U.S. Department of Commerce. 